\title{
Measured effect of collection and cooling conditions on the motility and the water transport parameters at subzero temperatures of equine spermatozoa
}

\author{
R. V. Devireddy ${ }^{1 *}$, D. J. Swanlund ${ }^{1,4}$, \\ A. S. Alghamdi², L. A. Duoos², M. H. T. Troedsson², \\ J. C. Bischof ${ }^{1,3,4}$ and K. P. Roberts ${ }^{4 \dagger}$ \\ ${ }^{1}$ Department of Mechanical Engineering, ${ }^{2}$ College of Veterinary Medicine, \\ ${ }^{3}$ Department of Biomedical Engineering, and ${ }^{4}$ Department of Urologic Surgery, \\ University of Minnesota, Minneapolis, MN 55455, USA
}

\begin{abstract}
The effects of extracellular ice and cryoprotective agents on the measured volumetric shrinkage response and the membrane permeability parameters of equine spermatozoa have been reported previously. The volumetric shrinkage data were obtained using a differential scanning calorimeter technique that was independent of cell shape. The aim of this study was to examine the effects of collection and cooling conditions on the motility and the water transport parameters at subzero temperatures of equine spermatozoa. Stallion semen samples were collected using either a commercial lubricating agent, which caused osmotic stress to the spermatozoa, or water-insoluble Vaseline ${ }^{\mathrm{T} M}$ as the artificial vagina lubricant. In some experiments, spermatozoa were cooled at $1^{\circ} \mathrm{C} \mathrm{min}^{-1}$ from $20^{\circ} \mathrm{C}$ to $4^{\circ} \mathrm{C}$ to induce cold shock. An Equitainer was used to achieve control cooling
\end{abstract}

rates $\left(\leqslant 0.3^{\circ} \mathrm{C} \mathrm{min}^{-1}\right)$ at temperatures $>0^{\circ} \mathrm{C}$. The water transport response of spermatozoa that were coldshocked and osmotically shocked was significantly different from that of control spermatozoa $(P<0.01)$. Osmotic stress appeared to have an effect on the water transport response, although this effect was not significant. These results indicate that cold shock alters the behaviour of equine spermatozoa in cryopreservation protocols as a result of changes in the water transport properties of the plasma membrane. Although osmotic stress did not significantly affect water transport in equine spermatozoa, it did significantly decrease sperm motility in the extended semen samples $(P<0.01)$, which would, in turn, lower the quality of cold-stored or cryopreserved spermatozoa.

\section{Introduction}

Although the first pregnancy obtained with frozen stallion semen was reported in 1957 by Barker and Gandier, effective and widespread use of frozen equine spermatozoa is still limited (Graham, 1996). Most understanding of the cryopreservation of equine spermatozoa (and mammalian spermatozoa in general) is still empirical, as the size and morphology of mammalian spermatozoa limit the applicability of standard cellular cryomicroscopy techniques to measure the biophysical response (water transport and intracellular ice formation) in spermatozoa during freezing. However, a recent advance in measurement methodology,

\footnotetext{
*Present address: Department of Mechanical Engineering, Louisiana State University, Baton Rouge, LA 70803, USA

${ }^{+}$Correspondence: Department of Urologic Surgery, University of Minnesota, 420 Delaware St S.E., MMC 394, Minneapolis, MN 55455, USA

Email: roberts@med.umn.edu
}

namely differential scanning calorimetry (DSC), has improved knowledge of the water transport response during freezing in stallion and other mammalian spermatozoa (Devireddy et al., 1999, 2000, 2002).

Stallion spermatozoa are extremely sensitive to cold shock (or 'chilling' injury) when cooled from $37^{\circ} \mathrm{C}$ to approximately $8^{\circ} \mathrm{C}$ at rates $\leqslant 0.3^{\circ} \mathrm{C} \mathrm{min}-1$. The cold shock effect produced by cooling rates $>0.3^{\circ} \mathrm{C} \mathrm{min}^{-1}$ includes abnormal patterns of swimming (circular or backwards), rapid loss of motility, acrosomal damage, plasma membrane damage, reduced metabolism and loss of intracellular components (Amann and Pickett, 1985; Moran et al., 1992). In addition, equine spermatozoa, like spermatozoa of several other species, are sensitive to osmotic excursion and permanently lose motility when exposed to both hyper- and hypo-osmotic stress (Ball and Vo, 2001). The aim of the present study was to investigate the changes, if any, that are induced in the permeability of the membrane to water during freezing as a result of collection conditions that cause osmotic shock, and suprazero cooling conditions that induce cold shock. 


\section{Materials and Methods}

\section{Semen collection and cooling}

Semen samples were obtained from three stallions housed at the University of Minnesota Veterinary Teaching Facility (St Paul, MN). One stallion was a 20-year-old Saddlebred (Legionnaire) and the other two were Arabians aged 6 (Chief) and 29 (Rooster) years. Gel-free ejaculates were collected using a Missouri artificial vagina. Priority Care 1 gel (First Priority Inc., Elgin, IL) was used in the artificial vagina to collect ejaculates in water-soluble lubricant. Vaseline ${ }^{\mathrm{TM}}$ (Cheesebrough Ponds, Greenwich, $\mathrm{CT}$ ) was used in the artificial vagina to collect ejaculates in water-insoluble lubricant. The osmolality of untreated semen from each stallion was determined using a freezepoint depression osmometer (Precision Systems Inc., Natick, MA).

After collection, untreated semen was diluted $1: 3$ in Kenneys skimmed milk extender (Har-Vet, Spring Valley, WI) at $37^{\circ} \mathrm{C}$ and slow cooled $\left(\leqslant 0.3^{\circ} \mathrm{C} \mathrm{min}{ }^{-1}\right)$ in an Equitainer-I ${ }^{T M}$ (Hamilton-Thorn, South Hamilton, MA). For experiments starting at room temperature (approximately $20^{\circ} \mathrm{C}$ ), samples were cooled to room temperature in an Equitainer and maintained at room temperature for the duration of the experiment, typically $4-6 \mathrm{~h}$. For experiments starting at $4^{\circ} \mathrm{C}$, samples were removed from the Equitainer $3-8 \mathrm{~h}$ after collection and maintained at $4^{\circ} \mathrm{C}$ for the duration of the experiment, typically $3-4 \mathrm{~h}$. The temperature of each sample was monitored using a digital thermometer with a K-type thermocouple (Fluke, Everett, WA). An aliquot of extended semen was kept at $37^{\circ} \mathrm{C}$ for determination of initial progressive motility and cell concentration using a computer-assisted semen analyser (Hamilton-Thorn).

Semen samples obtained using First Priority gel as the lubricant in the artificial vagina typically undergo osmotic shock as a result of contamination of the sample with the lubricant gel. The osmolality of the First Priority gel was approximately $1500 \mathrm{mOsm}$, as determined using a freezepoint depression osmometer. Therefore, samples collected using First Priority gel are referred to as 'osmotically shocked'. Control samples were collected using waterinsoluble Vaseline ${ }^{\mathrm{TM}}$ as the lubricant in the artificial vagina. Sperm suspensions were cooled at $1^{\circ} \mathrm{C} \mathrm{min}-1$ from $20^{\circ} \mathrm{C}$, the temperature at which the samples were removed from the Equitainer, to $4^{\circ} \mathrm{C}$ in the DSC sample pan, to examine the effect of cold shock or chilling injury on the water permeability of the sperm plasma membrane. These samples are referred to as 'cold shocked'. Water transport data on control samples that were not cold shocked or osmotically stressed, were taken from experiments performed at the same time and reported by Devireddy et al. (2002).

\section{Differential Scanning Calorimetry experiments}

DSC dynamic cooling experiments were performed on concentrated equine sperm samples in standard aluminium sample pans (Perkin Elmer Corporation, Norwalk, CT) in the presence of a lyophilized Pseudomonas syringae preparation (ATCC, Rockville, MD), a natural ice nucleator. In brief, $200 \mu \mathrm{l}$ of extended semen was concentrated by centrifugation at $400 \mathrm{~g}$ for $5 \mathrm{~min}$ at either room temperature or at $4^{\circ} \mathrm{C}$, and resuspended in approximately $25 \mu$ l of residual supernatant. Approximately $10 \mu \mathrm{l}$ of this sperm suspension was loaded in a DSC sample pan with approximately $0.5 \mathrm{mg}$ $P$. syringae. The concentration of the sperm suspension used in the DSC experiments ranged from $2 \times 10^{8}$ to $7 \times 10^{8}$ viable cells $\mathrm{ml}^{-1}$. The DSC dynamic cooling protocol used to measure the water transport out of equine spermatozoa is the same as that reported by Devireddy et al. (2002). A set of calibration and control experiments was performed as that described for a DSC-7 (Perkin Elmer Corp.) machine, to ensure the accuracy and repeatability of the experimental data (Devireddy and Bischof, 1998; Devireddy et al., 1998, 2002).

\section{Translation of heat release to cell volume data for dynamic cooling}

The heat release measured by the DSC, $\Delta q_{d s c}$ $\left(=q_{\text {initial }}-q_{\text {final }}\right)$ and $\Delta q(T)_{d s c}\left(=q(T)_{\text {initial }}-q(T)_{\text {final }}\right)_{\text {, is }}$ related to cell volume changes in cell suspensions by:

$$
\frac{V_{o}-V(T)}{V_{o}-V_{b}}=\frac{\Delta q(T)_{d s c}}{\Delta q_{d s c}}
$$

which can be rearranged to obtain water transport data as:

$V(T)=V_{o}-\frac{\Delta q(T) d s c}{\Delta q_{d s c}} \cdot\left(V_{o}-V_{b}\right)$

as described by Devireddy et al. (1998, 1999, 2000), where $V_{\mathrm{o}}$ is the isotonic volume of the equine spermatozoa and $V_{\mathrm{b}}$ is the osmotically inactive cell volume.

\section{Water transport model}

The reduction in cellular volume that occurs during freezing has been modelled thermodynamically (Levin et al., 1976; Mazur, 1984) and is described by the following relationship:

$$
\begin{aligned}
\frac{d V}{d T}= & -\frac{L_{p} A_{c} R T}{B v_{w}}\left[\ln \frac{\left(V_{o}-V_{b}\right)}{\left(V_{o}-V_{b}\right)+\varphi_{s} n_{s} v_{w}}\right. \\
& \left.-\frac{\Delta H_{f} v_{w} \rho}{R}\left(\frac{1}{T_{R}}-\frac{1}{T}\right)\right]
\end{aligned}
$$

where $L_{p}$ the permeability of the plasma membrane to water, is defined by:

$$
L_{p}=L_{p g} \exp \left(-\frac{E_{L p}}{R}\left(\frac{1}{T}-\frac{1}{T_{R}}\right)\right)
$$


Table 1. Comparison of initial seminal plasma osmolality and of sperm motility in extended seminal plasma samples from three stallions

\begin{tabular}{|c|c|c|c|c|}
\hline \multirow[b]{2}{*}{ Stallion } & \multicolumn{2}{|c|}{ Semen osmolality (mOsm) } & \multicolumn{2}{|c|}{ Sperm motility (\%) } \\
\hline & Priority One & Vaseline & Priority One & Vaseline \\
\hline Chief & $647^{* *}(44)$ & $302(2)$ & $44(13)$ & $55(7)$ \\
\hline Legionnaire & $534 * *(37)$ & $307(2)$ & $57 *(11)$ & $92(1)$ \\
\hline Rooster & $750^{* *}(36)$ & $307(2)$ & $39 *(10)$ & $83(2)$ \\
\hline Combined & $628^{* *}(30)$ & $305(1)$ & $48 *(7)$ & $75(4)$ \\
\hline
\end{tabular}

Each value represents analysis of a minimum of five ejaculates $(n \geqslant 5)$ from each stallion. Standard errors are shown in parentheses.

The value for Priority One is significantly different from the corresponding value for Vaseline at $* P<0.01$ or ${ }^{* *} P<0.001$.

where $L_{p g}$ is the reference membrane permeability at a reference temperature, $T_{\mathrm{R}}=273.15 \mathrm{~K} ; E_{L p}$ is the apparent activation energy $\left(\mathrm{kJ} \mathrm{mol}^{-1}\right)$ or the temperature dependence of the cell membrane permeability; $V$ is the sperm volume at temperature, $T(K) ; A_{c}$ is the effective membrane surface area for water transport, assumed to be constant during the freezing process; $V_{\mathrm{o}}$ and $V_{\mathrm{b}}$ are the isotonic and osmotically inactive sperm volumes, respectively. The equine spermatozoa was modelled as a cylinder $36.5 \mu \mathrm{m}$ in length and of radius $0.66 \mu \mathrm{m}$, which corresponds to a $V_{\mathrm{o}}$ of approximately $50 \mu \mathrm{m}^{3}$ and an $A_{\mathrm{c}}$ of approximately $150 \mu \mathrm{m}^{2}$ (Gravance et al., 1996; Gao et al., 1997). The osmotically inactive cell volume, $V_{\mathrm{b}}$, was assumed to be $0.6 V_{\mathrm{o}}$, a value within the range reported for a variety of mammalian spermatozoa (Gilmore et al., 1995; Willoughby et al., 1996; Gao et al., 1997); $R$ is the universal gas constant $=8.314$ $\mathrm{J} \mathrm{mol}^{-1} \mathrm{~K}$; $\mathrm{B}$ is the constant cooling rate, $\left(\mathrm{K} \mathrm{min}^{-1}\right) ; v_{w}$ is the molar volume of water $=18 \times 10^{12} \mu \mathrm{m}^{3} \mathrm{~mol}^{-1} ; \varphi_{\mathrm{s}}$ is the disassociation constant for salt, $=2 ; n_{\mathrm{s}}$ is the number of moles of salt, $C_{\mathrm{i}} \bullet\left(V_{\mathrm{o}}-V_{\mathrm{b}}\right)$, where $C_{\mathrm{i}}$ is the initial cell osmolality $=0.285 \mathrm{~mol} \mathrm{I}^{-1} ; \Delta H_{f}$ is the latent heat of fusion of water $=335 \mathrm{~mJ} \mathrm{mg}^{-1} ; \rho$ is the density of water $=1000 \mathrm{~kg} \mathrm{~m}^{-3}$. Several other assumptions are necessary to use equation [3] to model water transport in a cell system, as described by Devireddy et al. (2002).

\section{Numerical methods}

A non-linear least squares curve-fitting technique was used to calculate the values of $L_{p g}$ and $E_{L p}$ that best fit the volumetric shrinkage data (Bevington and Robinson, 1992; Devireddy et al., 1998). A detailed description of the curvefitting procedure has been described by Smith et al. (1998) and Devireddy et al. (2002).

\section{Results}

\section{Isolation of spermatozoa}

A total of 72 ejaculates obtained from three stallions was analysed for the effect of artificial vagina collection lubricant on neat semen osmolality, and on progressive motility of spermatozoa after a 1:3 dilution of semen in Kenneys extender. The mean osmolality of 20 semen samples collected using Priority Care 1 was significantly higher and much more variable than the osmolality of 52 semen samples collected using Vaseline (Table 1). In comparison with the use of Vaseline, the use of Priority Care 1 caused a marked increase in osmolality of semen from all the stallions from approximately $300 \mathrm{mOsm}$ to $750 \mathrm{mOsm}$ for Rooster, $647 \mathrm{mOsm}$ for Chief, and $534 \mathrm{mOsm}$ for Legionnaire. The effect of the artificial vagina lubricant on sperm motility is also shown (Table 1). Motility of spermatozoa from semen samples collected using Priority Care 1 was significantly lower than the motility of spermatozoa collected using Vaseline (47 versus $75 \%$ ). In comparison with the use of Vaseline, the use of Priority Care 1 had the most detrimental effect on the motility of spermatozoa from Rooster (a 53\% decrease), followed by Legionnaire (a 38\% decrease). Among the stallions, the motility of spermatozoa collected using Vaseline was poorest from Chief and not significantly different from spermatozoa collected using Priority Care 1. A scatter-plot of the relationship between semen osmolality and sperm motility is presented (Fig. 1). Spermatozoa collected using Priority Care 1 showed a moderately strong negative correlation $(r=-0.74)$ between increased osmolality and motility, whereas spermatozoa collected using Vaseline showed no correlation between these parameters.

\section{Dynamic cooling response and membrane permeability parameters}

A comparison of the water transport data at a cooling rate of $5^{\circ} \mathrm{C} \mathrm{min}{ }^{-1}$ for samples collected under various conditions of osmotic stress and cold shock is shown (Fig. 2). The bestfit parameters for $L_{p g}$ and $E_{L p}$ are shown (Table 2). The water transport parameters of control spermatozoa and spermatozoa that were osmotically stressed and cold shocked were significantly different from each other $(P<0.01)$, as measured using Student's $t$ test, over the entire temperature range of interest $\left(-0.53^{\circ} \mathrm{C}\right.$ to $\left.-15^{\circ} \mathrm{C}\right)$. Similarly, the differences in the water transport parameters between control spermatozoa and spermatozoa that were only cold shocked were also significant $(P<0.1)$, albeit within the narrower 


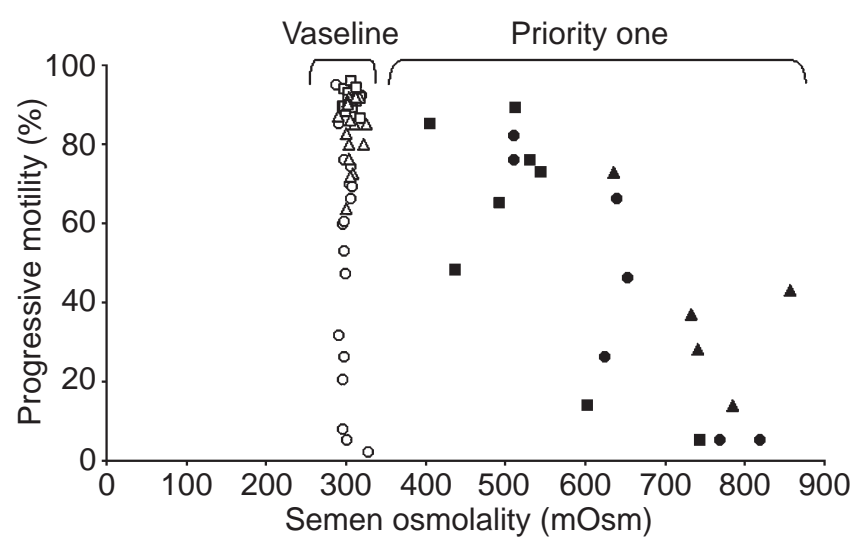

Fig. 1. Percentage of progressively motile equine spermatozoa from three stallions (Chief, Legionnaire and Rooster) at various initial seminal plasma osmolalities. All samples collected using a water-soluble lubricating gel (Priority one) in the artificial vagina had initial seminal plasma osmolalities $>400$ mOsm. Samples collected using Vaseline had initial seminal plasma osmolarities $<350 \mathrm{mOsm}$. A strong correlation between osmotic stress in the initial semen sample and decreased sperm motility in the extended suspension was apparent for samples collected using a watersoluble lubricant in the artificial vagina $(r=-0.74)$. Chief: sample collected using Vaseline $(\bigcirc)$ or water-soluble gel $(\bullet)$; Legionnaire: sample collected using Vaseline $(\square)$ or a water-soluble gel ( $\square$ ); Rooster: sample collected using Vaseline $(\triangle)$ or a water-soluble gel $(\mathbf{\Lambda})$.

temperature range of $-0.53^{\circ} \mathrm{C}$ and $-8.0^{\circ} \mathrm{C}$. Within this temperature range, $\geqslant 90 \%$ of the osmotically active intracellular water leaves the cell.

There were no significant differences in water transport parameters between control spermatozoa and spermatozoa that were only osmotically stressed. In addition, there were no significant differences in water transport responses between spermatozoa that were only cold shocked and spermatozoa that were both cold shocked and osmotically stressed. Taken together, these results indicate that cold shock is significantly detrimental to the water permeability characteristics of the plasma membrane of the spermatozoa, whereas osmotic stress, although very detrimental to sperm motility, has only a modest effect on water permeability characteristics of the sperm plasma membrane.

\section{Discussion}

The use of a water-soluble (First Priority) gel as a lubricant in the artificial vagina caused equine spermatozoa in the ejaculate to be rapidly and suddenly exposed to osmolalities as high as 800 mOsm; almost three times higher than the isotonic osmolality of 300 mOsm. A sudden and rapid increase in extracellular osmolality reduces sperm motility (Makler et al., 1981; Ball and Vo, 2001; Griggers et al., 2001). The effect of increased osmolality is one of the mechanisms of damage when spermatozoa are exposed to urine during retrograde ejaculation in humans or during

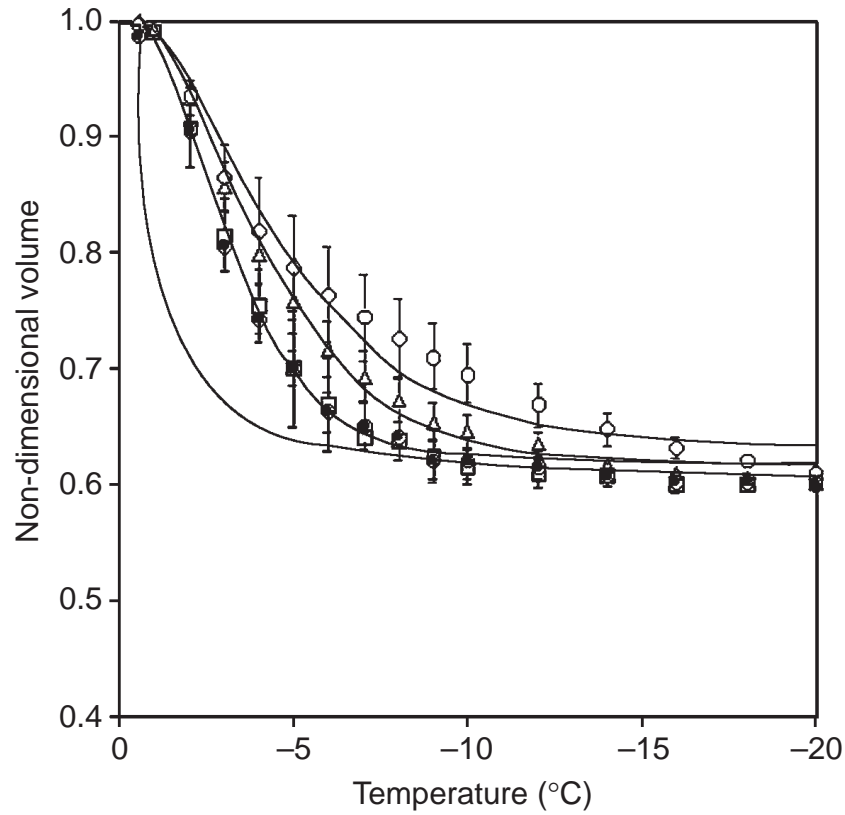

Fig. 2. Comparison of water transport data at $5^{\circ} \mathrm{C} \mathrm{min}^{-1}$ among control spermatozoa that were neither osmotically stressed nor cold shocked $(-$ ), spermatozoa that were osmotically stressed ( $\square$ ), spermatozoa that were cold shocked $(\triangle)$, and spermatozoa that were both osmotically stressed and cold shocked $(\bigcirc)$. Volumetric response of equine spermatozoa as a function of subzero temperatures was obtained using the differential scanning calorimeter technique. The model simulated dynamic cooling response is shown as a solid line and was obtained by using the 'best fit' membrane permeability parameters $\left(L_{p g}\right.$ and $\left.E_{L p}\right)$ at a cooling rate of $5^{\circ} \mathrm{C} \mathrm{min}{ }^{-1}$, in the water transport equation (Equations 3 and 4). The error bars represent the standard deviations in the data.

collection in stallions (Makler et al., 1981; Griggers et al., 2001). The loss of motility of stallion spermatozoa caused by hyperosmotic stress is permanent, with no recovery of motility upon return to iso-osmolality (Ball and Vo, 2001). This finding is consistent with the results of the present study in which motility of spermatozoa was measured after extension of the semen sample, which, in essence, measured the permanent motility loss incurred by acute hyperosmotic stress encountered by untreated semen at collection. These results indicate that the damage due to osmotic stress will contribute to compromised function of spermatozoa after cryopreservation. As water-soluble gel is the most common lubricant used in the artificial vagina for collecting stallion ejaculates, it is probable that a significant proportion of the stallion sperm samples collected in the field will experience osmotic damage during the collection procedure that will lead to variability in sperm motility.

These results of the present study also demonstrate that cold shock affects the water transport response and membrane permeability parameters of equine spermatozoa at subzero temperatures. Cold shock is related to phase transitions, such as an inverted hexagonal II phase, in the 
Table 2. Water transport parameters for equine spermatozoa in the presence of extracellular ice under various collection and cooling conditions at a cooling rate of $5^{\circ} \mathrm{C} \mathrm{min}^{-1}$

\begin{tabular}{lccc}
\hline Treatment & $L_{p g}\left(\mu \mathrm{m} \mathrm{min}^{-1} \mathrm{~atm}^{-1}\right)$ & $E_{L p}\left(\mathrm{kcal} \mathrm{mol}^{-1}\right)$ & $R^{2}$ value \\
\hline Control & 0.022 & 67.9 & 0.99 \\
Osmotic stress & 0.017 & 65.4 & 0.99 \\
Cold shock & $0.013^{*}$ & $57.4^{*}$ & 0.99 \\
Osmotic stress and cold shock & $0.01^{* *}$ & $54.5^{* *}$ & 0.98 \\
\hline
\end{tabular}

aData taken from Devireddy et al. (2002).

Value is significantly different from the control at $* P<0.1$ or ${ }^{* *} P<0.01$.

$L_{p g}$ : hydraulic permeability of the cell plasma membrane; atm: atmosphere; $E_{L p}$ : apparent activation energy.

sperm plasma membrane, which correlate with changes in the membrane permeability to ions such as $\mathrm{K}^{+}$and $\mathrm{Ca}^{2+}$ (Hammerstedt et al., 1990; Parks and Lynch, 1992; Drobnis et al., 1993; White, 1993). Loss of electrolyte homeostasis as a result of cold shock is, not surprisingly, detrimental to sperm function. Changes in the permeability of the sperm plasma membrane to water caused by cold shock, as shown in the present study, are also consistent with perturbation of the structure of the sperm plasma membrane. Osmotic shock contributes little to the change in membrane permeability to water, even though it markedly and permanently affects motility. This observation is consistent with the lack of change in the permeability of the equine sperm plasma membrane to propidium iodide after exposure to hyperosmotic solutions as high as $600 \mathrm{mOsm}$ (Ball and Vo, 2001). Thus, the effect of cooling at temperatures $>0^{\circ} \mathrm{C}$ on the water transport response and the predicted permeability parameters $\left(L_{p g}\right.$ and $\left.E_{L p}\right)$ appear to be more important than osmotic stress. However, both types of stress would be expected to affect the quality of spermatozoa after cryopreservation.

Finally, the present study demonstrates the variation in semen quality among stallions and also the differences in semen quality sometimes encountered among collections from the same stallion. All three stallions used in the present study displayed large variation in semen quality, as determined by motility of spermatozoa in extended semen samples, when collected using water-soluble gel as the lubricant in the artificial vagina. This variability was not apparent for two of the three stallions (Rooster and Legionnaire) when the water-insoluble lubricant was used for collection and illustrates a preventable cause of semen quality variation. However, semen quality from the third stallion (Chief) was variable regardless of the lubricant used for collection, indicating that semen quality of some stallions is variable for unknown reasons.

The present study demonstrates the first measured changes in the water transport response of equine spermatozoa in suspension as a result of variations in cooling and collection at temperatures $>0{ }^{\circ} \mathrm{C}$. Significant changes occurred in the plasma membrane of equine spermatozoa as a result of cold shock, and these differences were accentuated slightly by additional osmotic stress. It is well known that cold shock is detrimental to the function of equine spermatozoa, although an effect on membrane permeability to water during freezing had not been demonstrated previously. The results of the present study indicate that cold shock, in addition to direct damage to the spermatozoa, can affect water transport from the spermatozoa and, thereby, potentially alter the response of the spermatozoa to an established cryopreservation protocol. The present study also found that the water-soluble lubricant commonly used in the artificial vagina may cause an osmotic stress on the equine spermatozoa in the ejaculate, resulting in severe depression of sperm motility. This finding should be considered when equine semen is collected for intrauterine insemination or for use in cryopreservation protocols.

This work was supported by the Academic Health Center, University of Minnesota, Minneapolis, MN. The authors would like to thank N. Vetcher for her help with stallion semen collection.

\section{References}

Amann RP and Pickett BW (1987) Principle of cryopreservation and a review of cryopreservation of stallion spermatozoa Journal of Equine Veterinary Science 7 145-173

Ball BA and Vo A (2001) Osmotic tolerance of equine spermatozoa and the effects of soluble cryoprotectants on equine sperm motility, viability and mitochondrial membrane potential Journal of Andrology 22 1061-1069

Barker CAV and Gandier JCC (1957) Pregnancy in a mare resulted from frozen epididymal spermatozoa Canadian Journal of Comparative Medicine and Veterinary Science 21 47-51

Bevington PR and Robinson DK (1992) In Data Reduction and Error Analysis for the Physical Sciences pp 141-164. McGraw-Hill, New York

Devireddy RV and Bischof JC (1998) Measurement of water transport during freezing in mammalian liver tissue. Part II: The use of differential scanning calorimetry Journal of Biomechanical Engineering 120 559-569

Devireddy RV, Raha D and Bischof JC (1998) Measurement of water transport during freezing in cell suspensions using a differential scanning calorimeter Cryobiology 36 124-155

Devireddy RV, Swanlund DJ, Roberts KP and Bischof JC (1999) Subzero membrane permeability parameters of mouse spermatozoa in the presence of extracellular ice and cryoprotective agents Biology of Reproduction 61 764-775

Devireddy RV, Swanlund DJ, Roberts KP, Pryor JL and Bischof JC (2000) The effect of extracellular ice and cryoprotective agents on the water 
permeability parameters of human sperm plasma membrane during freezing Human Reproduction 15 1125-1135

Devireddy RV, Swanlund DJ, Olin T, Vincente W, Troedsson MHT, Bischof JC and Roberts KP (2002) Cryopreservation of equine spermatozoa: optimal cooling rates in the presence and absence of cryoprotective agents Biology of Reproduction 66 222-231

Drobnis EZ, Crowe LM, Berger T, Anchordoguy TJ, Overstreet JW and Crowe JH (1993) Cold shock damage is due to lipid phase transitions in cell membranes: a demonstration using sperm as a model Journal of Experimental Zoology 265 432-437

Gao DY, Mazur P and Critser JK (1997) Fundamental cryobiology of mammalian spermatozoa. In Reproductive Tissue Banking pp 263-328 Eds AM Karow and JK Critser. Academic Press, San Diego

Gilmore JA, McGann LE, Liu J, Gao DY, Peter AT, Kleinhans FW and Critser JK (1995) Effect of cryoprotectant solutes on water permeability of human spermatozoa Biology of Reproduction 53 985-995

Graham JK (1996) Cryopreservation of stallion spermatozoa Reproductive Technology 12 131-147

Gravance CG, Liu IKM, Davis RO, Hughes JP and Casey PJ (1996) Quantification of normal head morphometry of stallion spermatozoa Journal of Reproduction and Fertility 108 41-46

Griggers S, Paccamonti DL, Thompson RA and Eilts BE (2001) The effects of $\mathrm{pH}$, osmolarity and urine contamination on equine spermatozoal motility Theriogenology 56 613-622

Hammerstedt RH, Graham JK and Nolan JP (1990) Cryopreservation of mammalian sperm: what we ask them to survive Journal of Andrology 11 73-88

Levin RL, Cravalho EG and Huggins CG (1976) A membrane model describing the effect of temperature on the water conductivity of erythrocyte membranes at subzero temperatures Cryobiology 13 415-429
Makler A, David R, Blumenfeld Z and Better OS (1981) Factors affecting sperm motility. VII: sperm viability as affected by change of $\mathrm{pH}$ and osmolarity of semen and urine specimens Fertility and Sterility $\mathbf{3 6}$ 507-511

Mazur P (1984) Freezing of living cells: mechanisms and implications American Journal of Physiology 247 C125-C142

Moran DM, Jasko DJ, Squires EL and Amann RP (1992) Determination of temperature and cool rate induced cold shock in stallion spermatozoa Theriogenology 38 999-1012

Parks JE and Lynch DV (1992) Lipid composition and thermotropic phase behavior of boar, bull, stallion and rooster sperm membranes Cryobiology 29 255-266

Smith DJ, Schulte M and Bischof JC (1998) The effect of dimethylsulfoxide on the water transport response of rat hepatocytes during freezing American Society of Mechanical Engineers Journal of Biomechanical Engineering 120 549-558

White IG (1993) Lipids and calcium uptake of sperm in relation to cold shock and preservation: a review Reproduction, Fertility and Development 5 639-658

Willoughby CE, Mazur P, Peter AT and Critser JK (1996) Osmotic tolerance limits and properties of murine spermatozoa Biology of Reproduction 55 $715-727$

Received 22 March 2002.

First decision 27 May 2002

Revised manuscript received 5 June 2002.

Accepted 23 July 2002. 